This document is confidential and is proprietary to the American Chemical Society and its authors. Do not copy or disclose without written permission. If you have received this item in error, notify the sender and delete all copies.

\title{
Highly conductive silver nanoparticles functionalized aramid fiber paper for electric heaters with rapid response and chemical stability
}

\begin{tabular}{|r|l|}
\hline Journal: & Industrial \& Engineering Chemistry Research \\
\hline Manuscript ID & ie-2020-04005c.R2 \\
\hline Manuscript Type: & Article \\
\hline Complete List of Authors: & $\begin{array}{l}\text { Zhou, Yanfen; Qingdao University, } \\
\text { Sun, Zhenhua; Qingdao University, } \\
\text { Jiang, Liang; Qingdao University, } \\
\text { Chen, Shaojuan; Qingdao University } \\
\text { Ma, Jianwei; Qingdao University, } \\
\text { Zhou, Fenglei; University College London }\end{array}$ \\
\hline
\end{tabular}

\section{SCHOLARONE \\ Manuscripts}




\title{
Highly conductive silver nanoparticles functionalized aramid fiber
}

\section{paper for electric heaters with rapid response and chemical}

\author{
stability

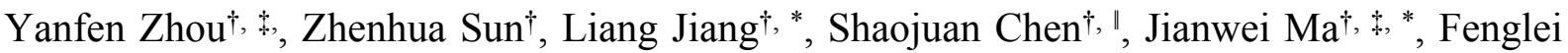 \\ Zhou ${ }^{\dagger}, \S$ \\ †College of Textiles and Clothing, Qingdao University, Qingdao, 266071, P. R. China \\ Industrial Research Institute of Nonwovens and Technical Textiles, Qingdao, 266071, P. R. \\ China \\ "Eco-Textile Collaborative Innovation Center, Qingdao University, Qingdao, 266071, P. R. \\ China \\ ${ }^{\S}$ Centre for Medical Image Computing, University College London, London, WC1V 6LJ, UK \\ *Corresponding authors. Email: liang.jiang@qdu.edu.cn(L. Jiang), mjwfz@qdu.edu.cn (J. Ma).
}




\begin{abstract}
Electrical heaters with rapid response, robust mechanical properties and reliable chemical stability are highly desirable to meet the requirements for the development of high performance heating systems that can be used in extreme conditions. Herein, highly conductive silver nanoparticles (AgNPs) functionalized aramid fiber paper (AFP) based electrical heaters were fabricated through an efficient plasma treatment and electroless plating method. The AFP/AgNPs shows an outstanding electrical conductivity with the electrical resistance as low as $0.047 \Omega / \mathrm{sq}$ and maintains high electrical conductivity after 20 washing cycles and 1000 bending cycles. The AFP/AgNPs based electrical heater could reach a temperature of about 210 ${ }^{\circ} \mathrm{C}$ at a low voltage of $1 \mathrm{~V}$ with a rapid response time of $20 \mathrm{~s}$. The electric heater also exhibited sufficient heating reliability, stability, and repeatability during long-term (up to $10000 \mathrm{~s}$ ) and 180 repeated heating and cooling cycles. Moreover, the electrical heaters maintain satisfying heating performances even after being treated with acid/alkali solutions and various organic solvents. The overall performance of AFP/AgNPs based electrical heaters has been demonstrated in the application of water heating and ice melting, indicating their great potential for high-performance heating systems that can be used in various harsh conditions.
\end{abstract}

Keywords: Aramid fiber paper; Silver nanoparticles; Plasma treatment; Electroless plating; Electrical heater.

\title{
1. Introduction
}

With the increasing demands for smart wearables, optoelectronics and temperaturepreservation device, electrical heaters which have light weight, high strength, ultra-flexibility and rapid response have received widespread attentions ${ }^{1-5}$. Electrical heaters which increase temperature by converting electrical energy into thermal energy can meet the requirements for different scenes such as smart clothing, defogging, ice melting and water heating ${ }^{6-9}$. Under the electrical voltage, the electrical current formed in the electrical heaters overcomes the resistance to generate Joule heat, which is in accordance with Joule's law as shown in the following 
equation:

$Q=I^{2} R t=U^{2} t / R$

Where $U$ is electrical voltage, $R$ is electrical resistance and $t$ is the heating time. According to Joule's law, a low electrical resistance is conducive to generating more Joule heat to reach the saturation temperature at a low supplied voltage, thereby achieving better heating performance $9,10$.

Traditionally, indium tin oxide (ITO) had been used extensively in various electrical heaters due to its good electrical conductivity and transparency ${ }^{11,12}$. However, ITO is carcinogenic (category 2B carcinogen), brittle and non-flexible, which greatly limits its applications, especially in smart clothing that is used in human bodies ${ }^{9,13}$. As an alternative, electrical heaters based on conductive polymer composites have attracted increasing concerns in recent years ${ }^{14-}$ 16. Various conductive components including carbon nanotubes (CNTs) ${ }^{17-20}$, graphene ${ }^{21-24}$, MXene ${ }^{25-27}$, metal nanoparticles/nanowires ${ }^{28-37}$ have been widely used in fabricating conductive polymer composites. Among various conductive components, silver has the highest conductivity $\left(1.65 \times 10^{-8} \Omega \cdot \mathrm{m}\right.$ of resistivity) and is preferred in fabricating conductive polymer composites for electrical heaters with high heating performances ${ }^{29-31,35}$.

Conductive polymer composites can be fabricated through two different methods, one of which is blending the conductive components with the polymer matrix ${ }^{24,38-41}$ and the other is coating the conductive components on the surface of various polymer substrates $20,25,42-46$. Electrical heaters based on blended conductive polymer composites had better long-term fastness ${ }^{19,21}$, whereas in contrast, electrical heaters prepared with surface functionalized polymers showed faster thermal response time due to the heat generated by and dissipated on the surface $5,18,26$, which achieved a balance between heat generation and heat dissipation rapidly.

Although there have been some progress on preparation of electrical heaters based on conductive polymer composites, it remains a challenge for electrical heaters to simultaneously 
achieve high temperature at low voltage, rapid response and high stability in extreme conditions. Aramid fiber paper (AFP), which is made by hot-press of high-performance pulp and short fiber, could act as an excellent substrate for fabricating high-performance paper-based electrical heaters owing to the advantages of high-strength, high-flexibility, high temperature resistance and excellent chemical stability ${ }^{47}$.

In the present study, we proposed a method for preparing a highly conductive silver nanoparticles (AgNPs) functionalized AFP (AFP/AgNPs) based electrical heater with rapidresponse and excellent chemical stability by combining low-temperature oxygen plasma treatment and electroless plating. The plasma treatment significantly enhances the interfacial interaction between the AFP substrate and the AgNPs, as well as the mechanical performance of the AgNPs functionlized AFP through the etching effect and the introduction of oxygencontaining groups. The electrical conductivity and heating performance of the AFP/AgNPs were investigated. The results show that the AFP/AgNPs has an outstanding electrical conductivity with the electrical resistance as low as $0.047 \Omega /$ sq. The AFP/AgNPs based electrical heaters exhibit rapid response, excellent stability and reliability upon long-term and repeated heating/cooling cycles as well as satisfying heating performances after being subjected to washing, bending deformation and chemical corrosion. The performance of the AFP/AgNPs composites based heater were demonstrated in water heating and deicing.

\section{Experimental}

\subsection{Materials}

Meta-aramid fiber papers with a thickness of $0.18 \mathrm{~mm}$ were supplied by ZhongFang Special Fiber Co., Ltd, Dongying, China. Ethanol, silver nitrate $\left(\mathrm{AgNO}_{3}\right)$, sodium hydroxide $(\mathrm{NaOH})$, ammonia $\left(\mathrm{NH}_{3} \cdot \mathrm{H}_{2} \mathrm{O}\right)$, glucose, sodium hydroxide $(\mathrm{NaOH})$, hydrochloric acid $(\mathrm{HCl})$, acetone, N-heptane and xylene were purchased from Sinopharm Chemical Reagent Co., Ltd, Shanghai, China. 


\subsection{Preparation of AFP/AgNPs Composites}

Initially, AFP were ultrasonically washed with $50 \mathrm{vol} \%$ of ethanol for 30 minutes to remove surface impurities and dried in vacuum at $60{ }^{\circ} \mathrm{C}$ for $2 \mathrm{~h}$. Then, the AFP were treated by using a plasma treater (AS 400, Plasmatreat $\mathrm{GmbH}$, Germany) at a nozzle speed of $1 \mathrm{~m} / \mathrm{min}$, a plasma cycle time of $80 \%$ and a nozzle height of $4-5 \mathrm{~cm}$. Immediately after the plasma treatment, the AFP were immersed in a ammoniacal silver nitrate solution $\left(5 \sim 30 \mathrm{~g} / \mathrm{L}\right.$ of $\mathrm{AgNO}_{3}$ concentration) for 30 minutes and then, glucose (with a concentration of $30 \mathrm{~g} / \mathrm{L}$ ) as a reducing agent was added to the ammoniacal silver nitrate solution at a dropping rate of about 60 drops/minute. The reaction was allowed to proceed at $30{ }^{\circ} \mathrm{C}$ for $24 \mathrm{~h}$. After electroless plating, the silver-coated MAFP was rinsed with deionized water three times and dried in vacuum oven at $60{ }^{\circ} \mathrm{C}$ for 24 hours. The AgNPs coated AFP obtained with $\mathrm{AgNO}_{3}$ concentration of 5, 10, 15, 20 and $30 \mathrm{~g} / \mathrm{L}$ was denoted as AFP/AgNPs-5, AFP/AgNPs-10, AFP/AgNPs-15, AFP/AgNPs-20, AFP/AgNPs-30, respectively.

\subsection{Characterization}

The surface morphology of AFP, plasma treated AFP and AFP/AgNPs was observed by using a scanning electron microscopy (SEM) (VEGA3, TESCAN, Czech). The surface element was scanned by using an energy dispersive spectrometer (EDS) (E1856-C2B, EDAX, USA). The chemical composition was investigated by using an X-ray photoelectron spectroscopy (XPS) (ESCALAB 250XI, Thermo Fisher Scientific, USA) with an Al Ka X-ray source (1486.6 eV photons). The thermal property of AFP, plasma treated AFP and AFP/AgNPs composite papers was studied by using a TG/DSC synchronous thermal analyzer (STA 449 F3, NETZSCH $\mathrm{GmbH}$, Germany). The measurement was conducted from 40 to $800{ }^{\circ} \mathrm{C}$ with a heating rate of $20{ }^{\circ} \mathrm{C} / \mathrm{min}$. The crystalline structure of the samples was investigated by using an $\mathrm{X}$-ray diffractometer (XRD, Rigaku Ultima IV, Japan) of $\mathrm{Cu} \mathrm{K \alpha}$ radiation with a wavelength of $1.54056 \AA$. The mechanical property of different AFP was investigated by using a universal 
tensile testing machine (Instron 5965, USA). The sheet resistance of AFP/AgNPs composite papers was measured by using a multifunction digital four-probe tester (ST-2258C, Suzhou Jingge Electronic Co., Ltd, China). For the heating performance tests, The AFP/AgNPs with a dimension of $60 \mathrm{~mm} \times 10 \mathrm{~mm}$ was mounted with conductive copper tape as the electrode. Silver paste was employed to connect the tiny gap between samples and the copper tape to guarantee good contact. Constant or repeatedly or gradiently changed voltages were applied to the electrical heaters by using a precision power supply (B2901A, Keysight, USA). The real-time surface temperature of the electrical heater was measured by using a contact thermometer (UT320A, Uni-Trend Technology Co. Ltd, China) and the infrared thermal images were captured by using an infrared thermal imager (TiS50, Fluke Electronic Instrumentation, USA).

\section{Results and discussion}

\subsection{Surface plasma treatment and silver plating of AFP}

Figure 1 illustrates the preparation process of AgNPs-coated AFP. Firstly, low temperature plasma treatment was employed to activate the surface of AFP. In order to evaluate the effect of plasma treatment, the surface morphology and chemical composition of AFP before and after plasma treatment was studied. Figure $2 \mathrm{a}$ and $\mathrm{b}$ show the SEM image of pristine AFP and plasma-treated AFP, respectively. It can be seen that different from pristine AFP (Figure 2a), large amounts of etching spots (as noted by the red cycles) appeared on the plasma-treated AFP surface (Figure 2b). EDS results (Figure S1, Supporting Information) further show that the content of oxygen element increased from $23.88 \%$ to $28.73 \%$ after plasma treatment, indicating more oxygen-containing groups in the surface of plasma-treated AFP. 


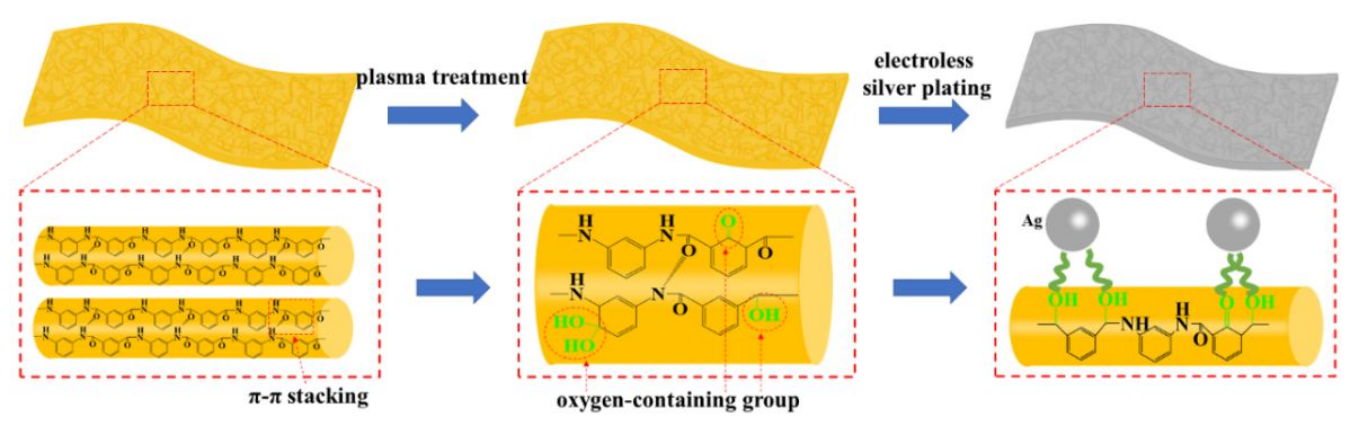

Figure 1. Schematic illustration for the fabrication of AgNPs functionalized AFP.

XPS was used to further study the effect of plasma treatment on the chemical composition of AFP. The XPS wide-scan spectra (Figure $2 \mathrm{~g}$ ) of pristine AFP and plasma-treated AFP showed three peaks at binding energy of about $285 \mathrm{eV}, 400 \mathrm{eV}$ and $532 \mathrm{eV}$ corresponding to $\mathrm{C} 1 \mathrm{~s}, \mathrm{~N}$ 1s and O 1s, respectively. The C 1s core-level spectrum of pristine AFP (Figure 2h) showed three peaks at binding energy of $284.4 \mathrm{eV}$ for $-\mathrm{C}-\mathrm{C}-/-\mathrm{C}-\mathrm{H}, 285.4 \mathrm{eV}$ for $-\mathrm{C}-\mathrm{N}-$ and $287.9 \mathrm{eV}$ for $-\mathrm{C}=\mathrm{O}$, which were ascribed to benzene ring and amide group in AFP. The $\mathrm{C}$ 1s core-level spectrum of plasma-treated AFP (Figure 2i) show two new peaks at binding energy of $286 \mathrm{eV}$ and $288.9 \mathrm{eV}$ belonging to $-\mathrm{C}-\mathrm{O}-$ and $-\mathrm{COO}-$, respectively, indicating that plasma treatment has introduced new oxygen-containing groups on the AFP surface. The N 1s core-level spectrum does not change after plasma treatment while the $\mathrm{O} 1 \mathrm{~s}$ core-level spectrum of plasma-treated AFP show two new peaks at binding energy of $530 \mathrm{eV}$ and $533 \mathrm{eV}$, which also confirms the introduction of oxygen-containing groups via plasma treatment (Figure S2, Supporting Information). Quantitative changes in chemical composition of the AFP before and after plasma treatment show that the content of carbon and nitrogen elements for AFP changed little after plasma treatment while the oxygen content varied obviously, with the $\mathrm{O} / \mathrm{C}$ ratio increased from 0.14 to 0.97 . The content of $-\mathrm{C}=\mathrm{O}$ increased from $4.37 \%$ to $7.56 \%$ and the contents of new formed -C-O- and -COO- bonds were $2.64 \%$ and $3.22 \%$, respectively. It is known that there is a strong $\pi-\pi$ conjugation in the molecular chain of aramid, which hinders the reactivity of the group ${ }^{5}$. The introduction of those oxygen-containing groups after plasma treatment is beneficial to break $\pi-\pi$ stacking balance and enhance the surface activity of AFP. 
After electroless silver plating (the $2^{\text {nd }}$ step in Figure 1), dense AgNPs deposited uniformly on the AFP surface as can be observed from the SEM images (Figure 2c) and the EDS mapping (Figure 2d) of AgNPs-coated AFP. The cross-sectional morphology (Figure 2e) shows that the silver coating adhered tightly to the AFP surface. The strong signals derived from Ag in the XPS wide-scan and Ag 3d core-level spectra of AFP/AgNPs in Figure $2 \mathrm{~g}$ demonstrate that AgNPs successfully deposited on the AFP surface. The Ag 3d core-level spectrum of AFP/AgNPs composite paper show two peaks at binding energy of $374 \mathrm{eV}$ for $\mathrm{Ag} 3 \mathrm{~d}_{3 / 2}$ and $368 \mathrm{eV}$ for $\mathrm{Ag} 3 \mathrm{~d}_{5 / 2}$, confirming that the silver particles were in the zerovalent state on the AFP substrate surface. The formation of dense AgNPs on the plasma-treated MAFP surface is due to the etching effect and the introduction of active oxygen-containing groups which could form ionic and covalent interacting with AgNPs ${ }^{48}$. As shown in Figure 2f, the LED bulbs connected in series and formed a logo of "QDU" that was lighted up when the AFP/AgNPs composite paper was connected with a power supply of $220 \mathrm{~V}$, indicating the high conductivity of the AgNPs coated AFP. 

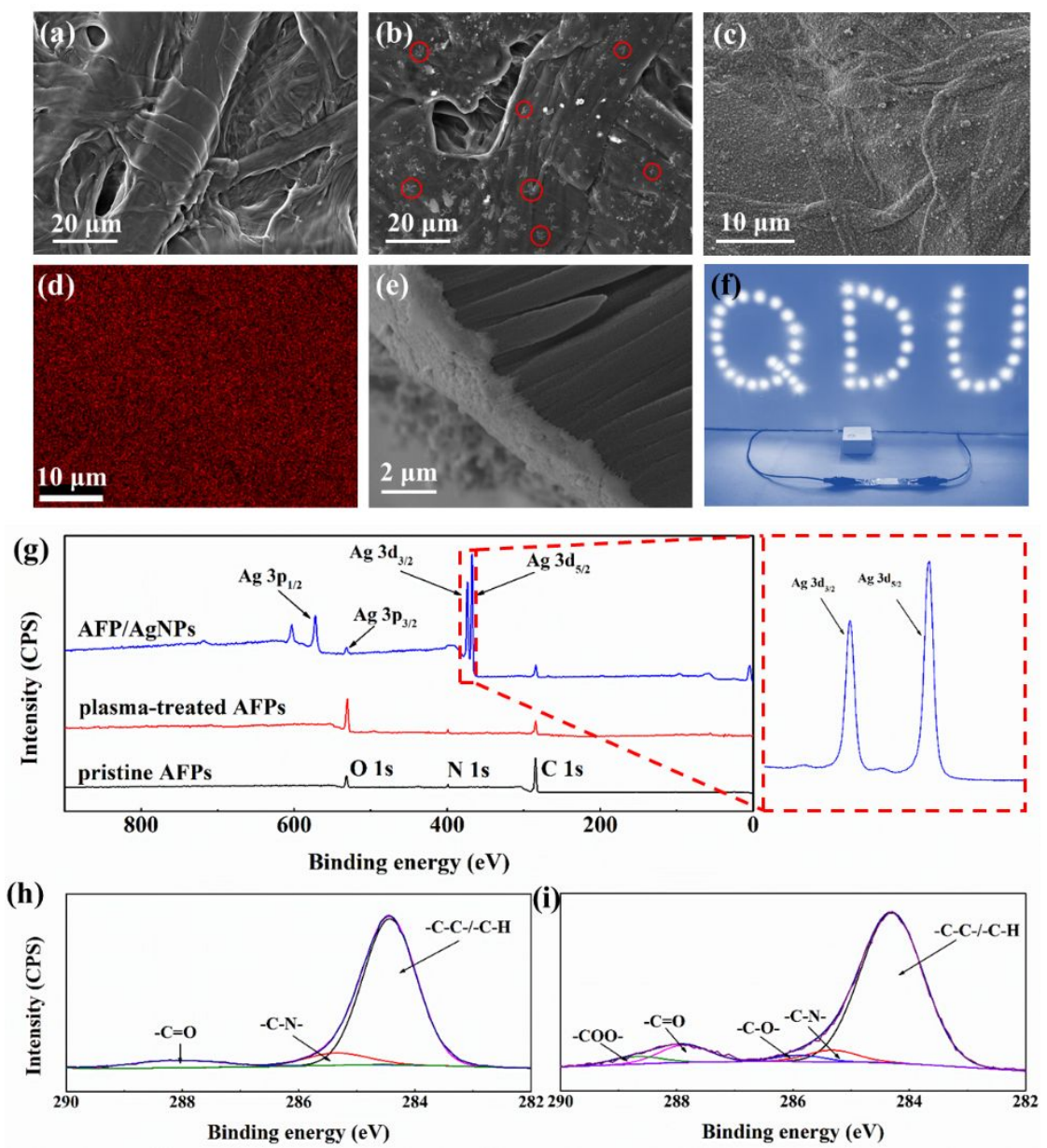

Figure 2. The SEM images of pristine AFP (a), plasma-treated AFP (the red cycles showing some of the etching pots) (b) and AgNPs-coated AFP (c); the EDS mapping of AgNPs-coated AFP (d); the cross-sectional morphology of AgNPs-coated AFP (e); lighting LED bulbs formed a logo of "QDU" when the AFP/AgNPs was connected with a power supply of $220 \mathrm{~V}$ (f); the XPS wide-scan spectra of pristine, plasma-treated and AgNPs-coated AFP (g); XPS spectra of C 1s peaks for pristine AFP (h) and plasma-treated AFP (i).

As a comparison, AgNPs-coated AFP which was not pre-treated with plasma was prepared, the SEM and EDS show that the AgNPs on the AFP surface was not continuous and no electrical conductivity was detected (Figure S3, Supporting Information).

\subsection{XRD Analysis, Electrical, Thermal and Mechanical Properties of AFP/AgNPs}

Figure 3a shows the XRD patterns of pristine AFP, plasma treated AFP and AgNPs coated AFP. The diffraction peak at the $2 \theta$ value of $27^{\circ}$, suggesting that the pristine AFP is a partly 
crystalline ${ }^{49}$. The crystal structure of AFP did not change after plasma treatment, indicating that the plasma treatment had no effect on the crystallinity of the AFP. Five new peaks at $2 \theta$ of $38.2^{\circ}, 44.4^{\circ}, 64.5^{\circ}, 77.5^{\circ}, 81.6^{\circ}$ corresponding to the (1 111$),\left(\begin{array}{lll}2 & 0 & 0\end{array}\right),\left(\begin{array}{lll}2 & 2 & 0\end{array}\right),\left(\begin{array}{lll}3 & 1 & 1\end{array}\right)$ and $(22$ 2) planes of face-centered cubic (FCC) lattice phase silver (JCPDS Card No.04-0783) appears on AgNPs-coated AFP. SEM images and EDS mapping of AFP/AgNPs prepared with variable $\mathrm{AgNO}_{3}$ concentration ranging from 5 to $30 \mathrm{~g} / \mathrm{L}$ show that the deposited silver layer for each sample is uniform and the surface silver content increases from $84.40 \%$ to $95.88 \%$ (Figure S4, Supporting Information). Figure $3 \mathrm{~b}$ shows the TGA curves of pristine, plasma-treated and AgNPs-coated AFP. It can be seen that the thermal degradation of all AFP samples is a twostep stage process. The $1^{\text {st }}$ stage in the temperature range of $40{ }^{\circ} \mathrm{C}$ to $200{ }^{\circ} \mathrm{C}$ was due to the evaporation of bound water contained in the material and the $2^{\text {nd }}$ stage starting from $430{ }^{\circ} \mathrm{C}$ was attributed to partial dehydroxylation and alkoxide decomposition. These results indicate that the thermal stability of AFP samples is good and is not apparently influenced by plasma treatment and chemical deposition of AgNPs.

The relationship between $\mathrm{AgNO}_{3}$ concentration and electrical sheet resistance (Figure 3c) shows that as the $\mathrm{AgNO}_{3}$ concentration increases, the sheet resistance decreases sharply from $0.439 \Omega / \mathrm{sq}$ to about $0.076 \Omega / \mathrm{sq}$ when the $\mathrm{AgNO}_{3}$ concentration increases from 5 to $15 \mathrm{~g} / \mathrm{L}$ and then decreases slightly to $0.047 \Omega / \mathrm{sq}$ when the $\mathrm{AgNO}_{3}$ concentration increase further to $30 \mathrm{~g} / \mathrm{L}$. The stability of AgNPs coating on AFP was evaluated by measuring the electrical conductivity after bending and washing. Figure $3 \mathrm{~d}$ shows the change in sheet resistance of AFP/AgNPs-20 as an example with bending and washing cycles. It can be seen that the sheet resistance of AFP/AgNPs-20 increases from $0.049 \Omega /$ sq to $0.11 \Omega /$ sq after 1000 bending cycles and to 0.13 $\Omega /$ sq after 20 washing cycles. The above results suggest that the AFP/AgNPs have reliable bending and washing fastness.

Mechanical properties are of great importance considering the practical applications of AgNPs- 
coated AFP. Figure 3e and $3 \mathrm{f}$ shows the typical stress-strain curves and the average tensile strength and elongation at break of pristine, plasma-treated and AgNPs-coated AFP, respectively. It can be seen that compared with pristine AFP, the tensile strength and elongation at break of plasma treated AFP increased by $16.1 \%$ and $115.4 \%$, respectively. This was due to the fact that the increasing surface roughness and content of active oxygen-containing groups enhanced the bonding strength between adjacent fibers ${ }^{50,51}$. Interestingly, there are no obvious changes in both tensile strength and elongation at break between plasma treated AFP with and without AgNPs coating.
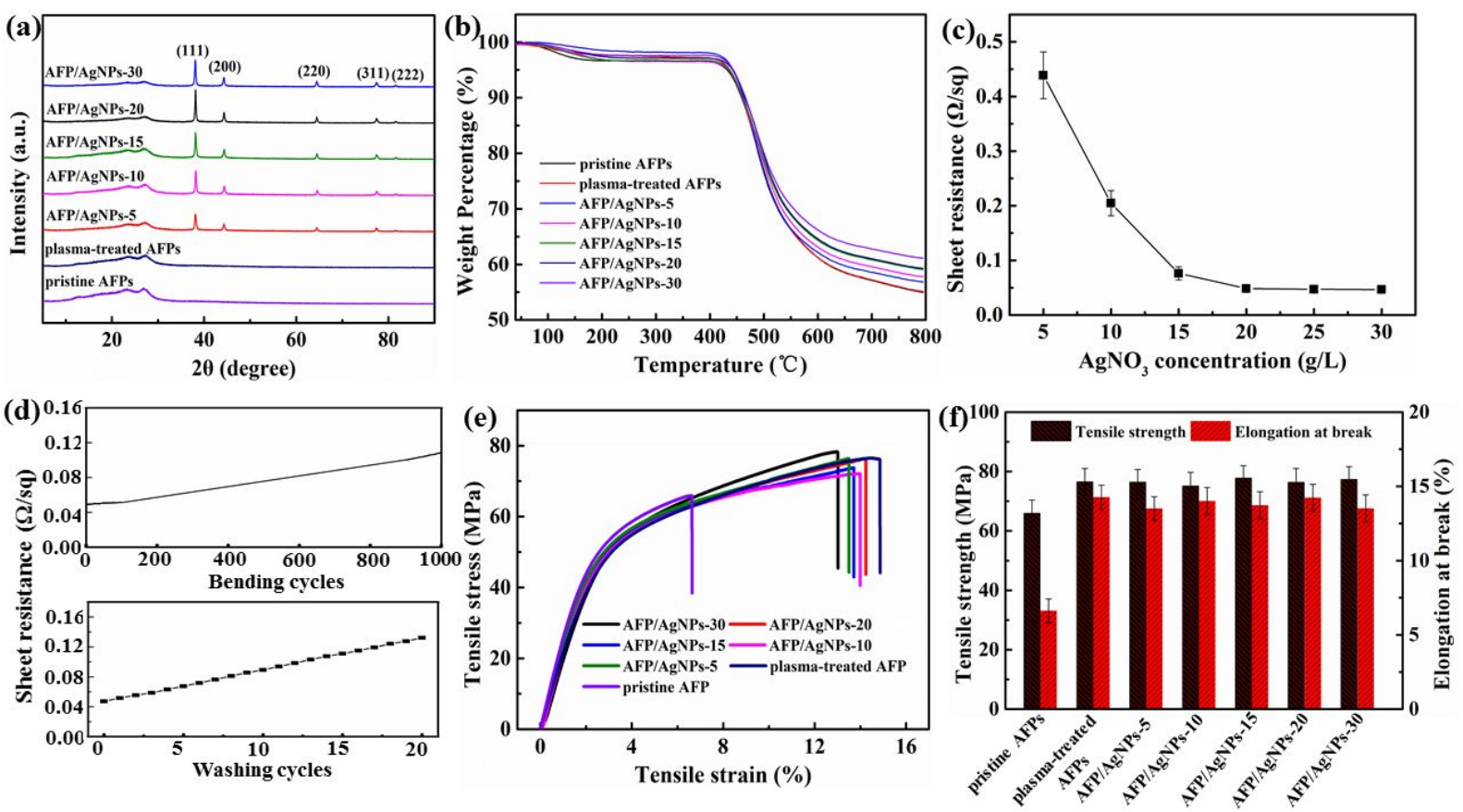

Figure 3. The XRD patterns of pristine AFP, plasma-treated AFP and AFP/AgNPs (a); the TGA curves of pristine AFP, plasma-treated AFP and AFP/AgNPs (b); the relationship between $\mathrm{AgNO}_{3}$ concentration and sheet resistance (c); the variation of sheet resistance of AFP/AgNPs20 with bending and washing cycles (d); the stress-strain curves (e) and the tensile strength and elongation at break (f) of pristine AFP, plasma-treated AFP and AFP/AgNPs.

\subsection{Heating performance of AFP/AgNPs}

Figure $4 \mathrm{a}$ and $\mathrm{b}$ shows the time-dependent temperature under different supplied voltages for the two electrical heaters based on AFP/AgNPs-5 and AgNPs-30, respectively. It can be seen that 
for both electrical heaters, the saturation temperature $(T s)$ increases with increasing supplied voltage and is reached within $20 \mathrm{~s}$, demonstrating their rapid response; When the power was switched off, the heater temperature decreases to room temperature within $25 \mathrm{~s}$, demonstrating equally rapid cooling response. At a supplied voltage of $1 \mathrm{~V}$, the electrical heater base on AFP/AgNPs-30 reached a saturation temperature of around $210{ }^{\circ} \mathrm{C}$, which is higher than other silver-coated polymer composites ${ }^{1-3,52-54}$.

Figure $4 \mathrm{c}$ shows the experimental data and the linear fittings of saturation temperature versus applied voltage. When the voltage is supplied to the electrical heater, the electrical energy is converted into thermal energy and the heater temperature rises rapidly; this process is companied by the heat generation of the electrical heater and heat radiation to the surrounding environment. According to the energy balance principle, the surface temperature of electrical heaters would reach an equilibrium when the dissipated power by Joule heating is equal to the power losses through conduction, convection, and radiation ${ }^{34}$. Accordingly, the surface temperature of electrical heaters could be calculated by using the following equation:

$$
\frac{U^{2}}{R}=m c \frac{d T}{d t}+h A\left(T-T_{0}\right)
$$

Where $U$ is the supplied voltage, $R$ is the electrical resistance of electrical heater, $m$ is the mass of the electrical heater, $c$ is the specific heat capacity, $h$ is the convective heat-transfer coefficient, $A$ is the area of electrical heater, $T$ is the surface temperature and $T_{0}$ is the initial ambient temperature. By integrating Eqn. 2 and taking $T(t=0)=T_{0}$, the time-dependent surface temperature and $T s$ of electrical heaters could be obtained as shown below ${ }^{5}$ :

$$
\begin{aligned}
& T=T_{0}+\frac{U^{2}}{R h A}\left(1-e^{-(h A / m c) t}\right) \\
& T_{S}=T_{0}+\frac{U^{2}}{R h A}
\end{aligned}
$$

It can be seen from Figure $4 \mathrm{c}$ that the saturation temperature of heaters shows an excellent linear relationship with $U^{2}$, suggesting that the saturation temperature achieved at various supplied 
voltages can be accurately calculated by the linear fitting within the materials range.

Aramid fiber paper, as a high-performance substance, would also be thermally decomposed from about $430{ }^{\circ} \mathrm{C}$ as indicated by the TGA measurement shown in Figure 3b. It can be seen from Figure 4d that at a certain applied voltage, the heaters temperature reaches the highest and then drops to zero, indicating the heater didn't function anymore. The applied voltage at which the temperature starts to drop is $3.2 \mathrm{~V}$ and $2.1 \mathrm{~V}$ for AFP/AgNPs-5 and AFP/AgNPs-10 based heater, respectively and about $1.8 \mathrm{~V}$ for AgNPs-15, AFP/AgNPs-20 and AFP/AgNPs-30 based heaters. It can also be noted that the highest heating temperature at which all heaters stopped function is around $430{ }^{\circ} \mathrm{C}$, which is in consistent with the TGA results (Figure $3 b$ ).

Figure 4e shows the change in heating temperature upon the gradient voltages for AFP/AgNPs5 and AFP/AgNPs-30 based electrical heaters. It can be seen that as the voltage gradually changes, the temperature of two heaters rapidly increases or decreases, suggesting the rapid and controlled response of the heater to temperature by simply tuning the applied voltage. The heating repeatability and long-term heating stability of AFP/AgNPs based electrical heater were investigated by using AFP/AgNPs -30 as an example. It can be seen from Figure $4 \mathrm{f}$ that when the electrical heater is subjected to an alternating voltage of $0 \mathrm{~V}$ and $1 \mathrm{~V}$, the heater shows simultaneous temperature change between room temperature $\left(23.7^{\circ} \mathrm{C}\right)$ and about $210{ }^{\circ} \mathrm{C}$. Moreover, there is no change in the heating/cooling behaviors of the heaters throughout the repeated on/off voltage for $10000 \mathrm{~s}$ (180 cycles), demonstrating the excellent repeatability of the electrical heaters. The stability of electrical heaters was also investigated by applying a constant supplied voltage. It can be seen from Figure $4 \mathrm{~g}$ that at a constant supplied voltage of $0.5 \mathrm{~V}$, the $T s$ of the heaters based on AFP/AgNPs-30 is very stable at around $115^{\circ} \mathrm{C}$ for the test period of $10000 \mathrm{~s}$, indicating good long-term stability of the heater. 

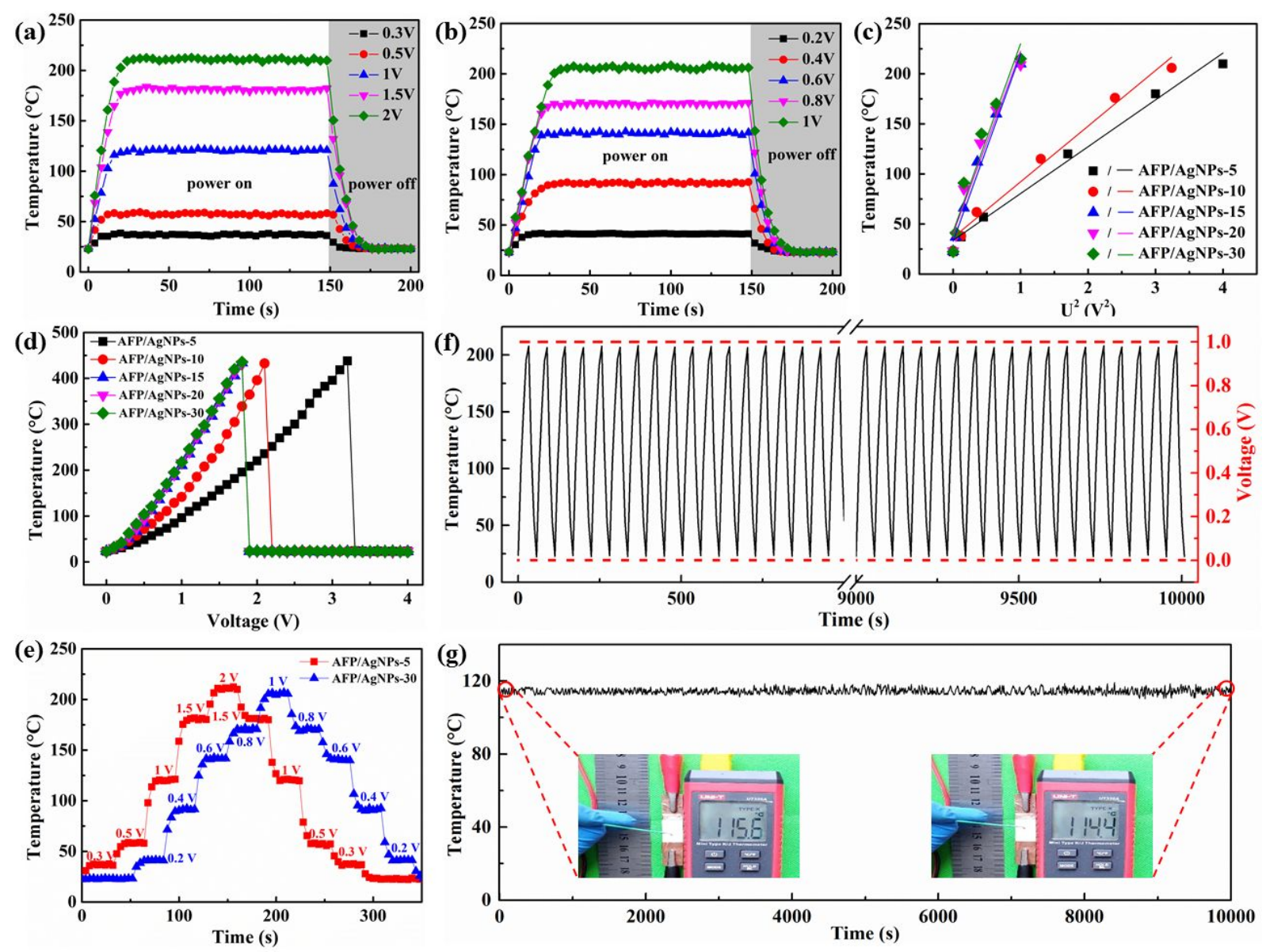

Figure 4. Time-dependent temperature at different supplied voltage for two electrical heaters based on AFP/AgNPs-5 (a) and AFP/AgNPs-30 (b); the linear fitting of saturation temperature versus $U^{2}(\mathrm{c})$; the relationship between surface temperature and voltage for $\mathrm{AFP} / \mathrm{AgNPs}$ electrical heaters (d); surface temperature of electrical heaters based on AFP/AgNPs-5 and AFP/AgNPs-30 upon gradiently changed voltages (e); the heating repeatability upon alternating voltage of $0 \mathrm{~V} / 1 \mathrm{~V}$ (f) and the long-term stability upon a supplied voltage of $0.5 \mathrm{~V}$ (g) for electrical heaters based on AFP/AgNPs-30.

Electrical heaters are inevitably subjected to various deformations and harsh environmental conditions during practical application. Therefore, it is important to evaluate the heating and cooling behaviors of the developed heaters in these conditions. Here the electrical heater prepared from AFP/AgNPs-15 was used as an example to demonstrate its stability in various extreme conditions.

Figure 5a-c show the heating performance of the electrical heaters before and after 1000 
bending cycles or 20 washing cycles. It can be seen from Figure 5a that after bending or washing, the saturation temperature of the electrical heater remains nearly unchanged, as revealed by the fact all heaters reached around $100^{\circ} \mathrm{C}$ within $20 \mathrm{~s}$ at a supplied voltage of $0.5 \mathrm{~V}$. It can be seen from Figure 5b-c that the electrical heater after 1000 bending cycles or 20 washing cycles still demonstrates good stability at a constant voltage of $0.5 \mathrm{~V}$. In addition, the electrical heaters after bending or washing also show excellent repeatability under the alternating voltage of 0 $\mathrm{V} / 1 \mathrm{~V}$. The digital and infra-red (IR) images in Figure 5d show the performance of the flexible electrical heaters based on AFP/AgNPs-15 in the "straight" and "bent" states with a supplied voltage of $0.5 \mathrm{~V}$. It can be seen that the electrical heater reaches $102.4{ }^{\circ} \mathrm{C}$ with a supplied voltage of $0.5 \mathrm{~V}$ in straight state and the saturation temperature remains nearly unchanged in the "bent" state $\left(101.1{ }^{\circ} \mathrm{C}\right)$, indicating its feasibility to be used as a flexible electrical heater candidate.

To evaluate the chemical stability of the AFP/AgNPs based electrical heater, the AFP/AgNPs15 were immersed in acid solution $(\mathrm{pH}=2)$, neutral aqueous solution $(\mathrm{pH}=7)$, alkali solution $(\mathrm{pH}=12)$ and various organic solvents including ethanol, acetone, $\mathrm{N}$-heptane and xylene for 3 days, the electrical conductivity of AFP/AgNPs-15 and heating performance of the electrical heaters based on the treated AFP/AgNPs-15 were evaluated. It can be seen from Figure 5e that after being immersed in the above mentioned solutions and organic solvents for 3 days, the sheet resistance of AFP/AgNPs-15 increased by $12.4 \%, 5.6 \%, 3.6 \%, 24.2 \%, 1.6 \%, 2.5 \%$ and $7.0 \%$, respectively, and the saturation temperature of AFP/AgNPs-15 based electrical heater decreased by $6.9 \%, 2.1 \%, 6.4 \%, 11.3 \%, 5.4 \%, 3.5 \%$ and $4.2 \%$, respectively. It can be seen from Figure $5 \mathrm{f}$ that the time-dependent temperature changes for electrical heaters prepared from treated AFP/AgNPs-15 appear to be similar to that before immersion, and the response time is still within $20 \mathrm{~s}$. Figure S5 (Supporting Information) shows the repeatability with pulse voltage of $0 \mathrm{~V} / 1 \mathrm{~V}$ and long-term stability with constant voltage of $0.5 \mathrm{~V}$ for electrical heaters after 
immersion. It can be seen that all electrical heaters with treated AFP/AgNPs-15 maintain excellent repeatability over hundreds of on/off voltage cycles and good long-term heating stability with a supplied voltage of $0.5 \mathrm{~V}$ for $10000 \mathrm{~s}$. The above results demonstrate that the AFP/AgNPs based electrical heater has excellent chemical resistance. The corrosion resistance might be related with the enhanced surface roughness and hence the hydrophobicity of $\mathrm{AFP} / \mathrm{AgNPs}$ (the water contact angle of AFP/AgNPs-15 is $106^{\circ}$ ), which to a certain degree, prevent the water or even the corrosion solution diffusing inside the material ${ }^{55,56}$. Besides, the integrity of the silver coating layer is well preserved after immersion (Figure S6, Supporting Information) due to the enhanced interfacial interaction between AgNPs and AFP contributed by plasma treatment.
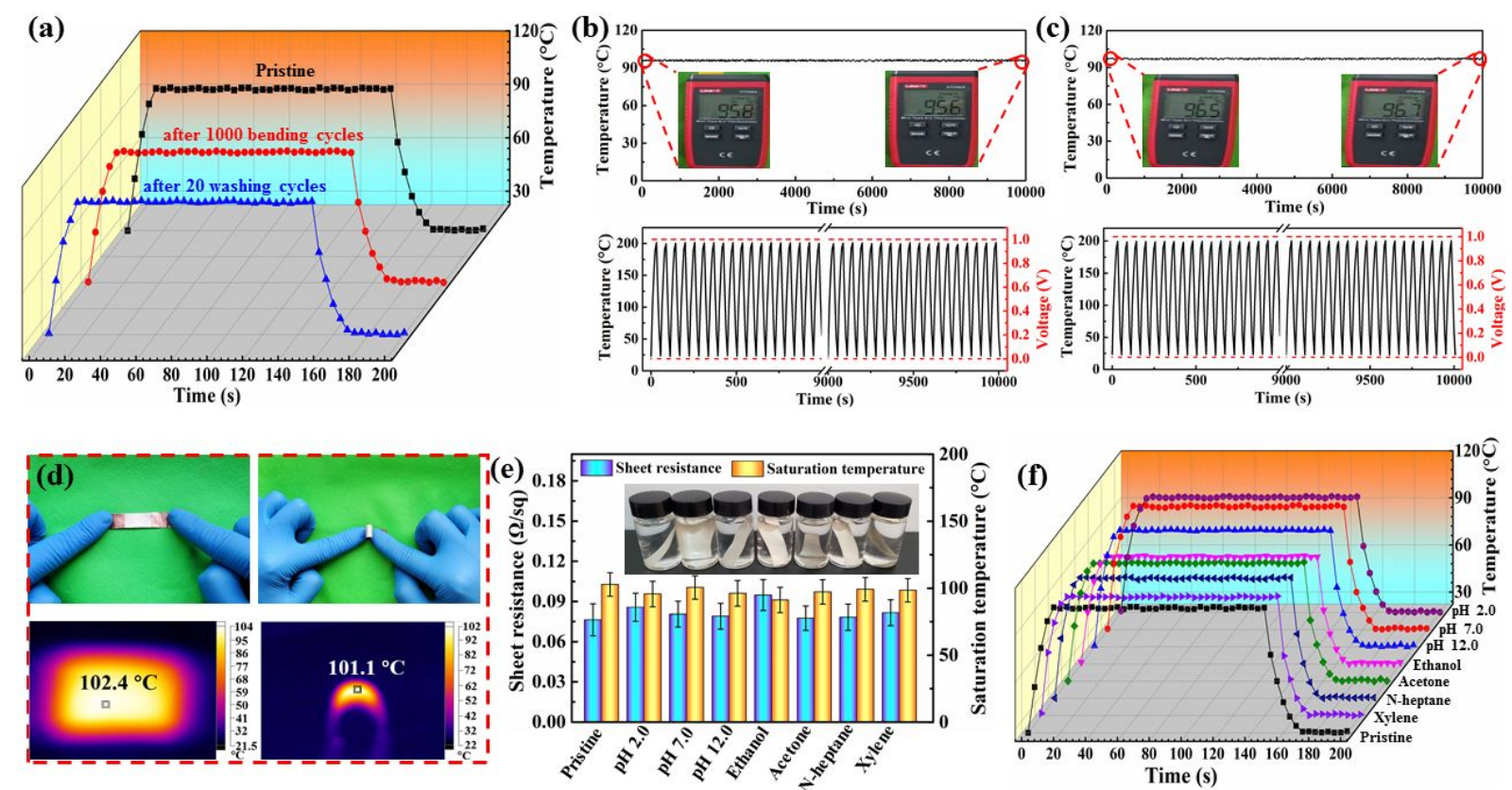

Figure 5. Time-dependent temperatures of electrical heaters based on AFP/AgNPs-15 after 1000 bending cycles and 20 washing cycles (a); the long-term stability and repeatability of electrical heaters after 1000 bending cycles (b) and 20 washing cycles (c); the digital and IR images of electrical heater in "straight" and "bent" state with supplied voltage of $0.5 \mathrm{~V}(\mathrm{~d})$; the sheet resistance and saturation temperature of electrical heaters based on AFP/AgNPs-15 after soaking for 3 days (e) and time-dependent temperatures of electrical heaters after soaking for 3 
days (f).

\subsection{Applications of the AFP/AgNPs based electrical heater in water heating and ice melting}

Figure 6a shows the use of an AFP/AgNPs-15 based heater to heat water. The electrical heater sample with an area size of $2 \mathrm{~cm} \times 2 \mathrm{~cm}$ was placed at the bottom of a glass bottle containing $20 \mathrm{ml}$ deionized water. The water was heated from $25{ }^{\circ} \mathrm{C}$ to $57{ }^{\circ} \mathrm{C}$ within $15 \mathrm{~min}$ upon the supplied voltage of $1 \mathrm{~V}$ and then reached $84{ }^{\circ} \mathrm{C}$ after another $15 \mathrm{~min}$. Figure $6 \mathrm{~b}$ shows the ice melting by using AFP/AgNPs-15 based heater. When an ice cube with a weight of 10 grams was left at room temperature without the heaters, it took as long as $50 \mathrm{~min}$ for the ice cube to melt completely. Whereas in contrast, when an ice cube with the same weight was placed on the top of the AFP/AgNPs-15 based heater with a size of $2 \mathrm{~cm} \times 2 \mathrm{~cm}$, the ice cube melt completely in only 9 min at a supplied voltage of $0.5 \mathrm{~V}$. The above results demonstrate that the AFP/AgNPs based electrical heater has great potential for application in low-voltage and highefficiency water heating and ice melting.
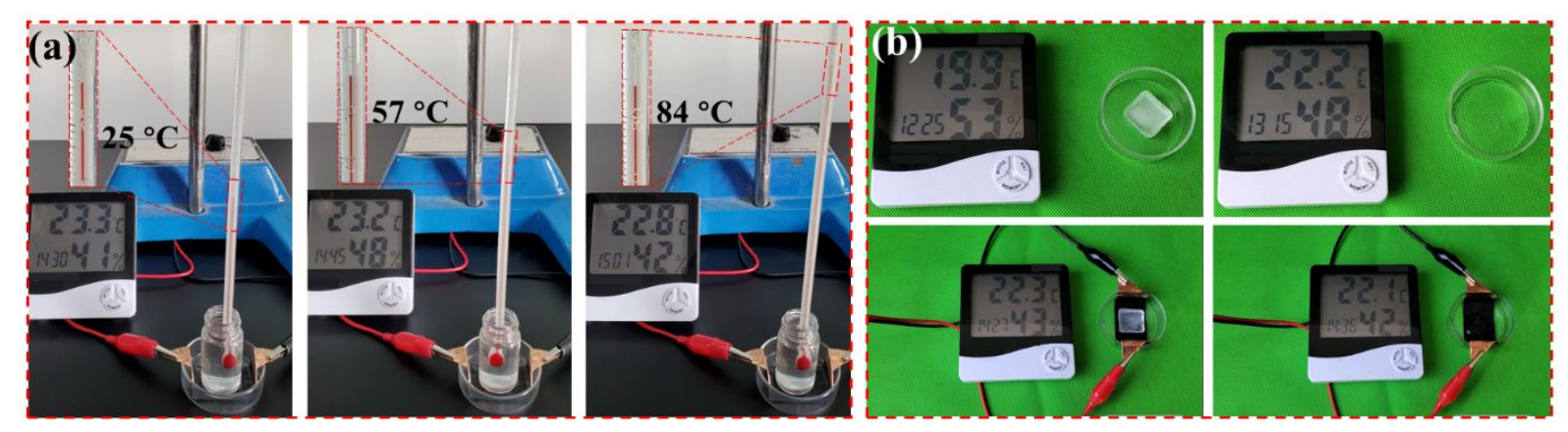

Figure 6. The digital images of electrical heaters in water heating with a voltage of $1 \mathrm{~V}$ (a) and ice melting with a voltage of $0.5 \mathrm{~V}(\mathrm{~b})$.

\section{Conclusions}

In conclusion, this work has reported the fabrication and performance of high-performance AgNPs functionalized AFP based electrical heaters through a very simple approach combining low-temperature oxygen plasma treatment and chemical deposition. The plasma treatment greatly favored the efficient deposition of AgNPs on the AFP surface through the etching effect and the introduction of oxygen-containing groups. The as-prepared AgNPs functionalized AFP 
showed excellent electrical conductivity with the electrical resistance as low as $0.047 \Omega /$ sq and maintained high electrical conductivity after 20 washing cycles or 1000 bending cycles. The electrical heater based on AFP/AgNPs reached a high temperature of about $210^{\circ} \mathrm{C}$ at a low voltage of $1 \mathrm{~V}$ and showed rapid response to the change in applied voltage. The electric heater also possessed sufficient heating reliability, stability, and repeatability during long-term and repeated heating and cooling cycles. In addition, the electrical heater exhibited outstanding heat performance after being treated with acid/alkali solutions and various organic solvents. The excellent heating performance of the AFP/AgNPs based electrical heater was demonstrated by water heating and ice melting. It can be envisaged that the electrically conductive AFP is a promising candidate for developing high performance flexible heating devices for applications in extreme conditions.

\section{Acknowledgements}

The authors would like to thank the financial support from the Shandong Provincial Key Research and Development Program, China (Grant no. 2019GGX102071, 2018GGX108003), the National Key Research and Development Program of China (Grant no. 2017YFB03098052), the Shandong "Taishan Youth Scholar Program" and the National Natural Science Foundation of China (Grant no. 51703108).

\section{Appendix A. Supplementary data}

SEM images and EDS results of pristine AFP and plasma-treated AFP; the XPS N 1s and O 1s spectra peaks for pristine AFP and plasma-treated AFP; the electrical resistance, SEM image and EDS mapping of AgNPs-coated AFP without plasma treatment; the SEM image and EDS mapping of silver-coated AFP obtained with different $\mathrm{AgNO}_{3}$ concentration; heating repeatability and long-term stability of AFP/AgNPs based electrical heater after being soaked in acid/alkali solutions and various organic solvents; SEM image of AFP/AgNPs-15 after being immersed in acid/alkali solutions and various organic solvents. 


\section{References}

1. Hong, S.; Lee, H.; Lee, J.; Kwon, J.; Han, S.; Suh, Y. D.; Cho, H.; Shin, J.; Yeo, J.; Ko, S.

H., Highly Stretchable and Transparent Metal Nanowire Heater for Wearable Electronics Applications. Advanced Materials 2015, 27 (32), 4744-4751.

2. Jo, H. S.; Kwon, H. J.; Kim, T. G.; Park, C. W.; An, S.; Yarin, A. L.; Yoon, S. S., Wearable transparent thermal sensors and heaters based on metal-plated fibers and nanowires. Nanoscale 2018, 10 (42), 19825-19834.

3. Lee, C. H.; Yun, Y. J.; Cho, H.; Lee, K. S.; Park, M.; Kim, H. Y.; Son, D. I., Environmentfriendly, durable, electro-conductive, and highly transparent heaters based on silver nanowire functionalized keratin nanofiber textiles. Journal of Materials Chemistry C 2018, 6 (29), 7847 7854.

4. Huang, Y.; Tian, Y.; Hang, C.; Liu, Y.; Wang, S.; Qi, M.; Zhang, H.; Zhao, J., Self-Limited Nanosoldering of Silver Nanowires for High-Performance Flexible Transparent Heaters. ACS Applied Materials \& Interfaces 2019, 11 (24), 21850-21858.

5. Ma, Z.; Kang, S.; Ma, J.; Shao, L.; Wei, A.; Liang, C.; Gu, J.; Yang, B.; Dong, D.; Wei, L.; Ji, Z., High-Performance and Rapid-Response Electrical Heaters Based on Ultraflexible, Heat-Resistant, and Mechanically Strong Aramid Nanofiber/Ag Nanowire Nanocomposite Papers. ACS Nano 2019, 13 (7), 7578-7590.

6. Jang, N. S.; Kim, K. H.; Ha, S. H.; Jung, S. H.; Lee, H. M.; Kim, J. M., Simple Approach to High-Performance Stretchable Heaters Based on Kirigami Patterning of Conductive Paper for Wearable Thermotherapy Applications. ACS Applied Materials \& Interfaces 2017, 9 (23), 19612-19621.

7. Lordan, D.; Burke, M.; Manning, M.; Martin, A.; Amann, A.; O’Connell, D.; Murphy, R.; Lyons, C.; Quinn, A. J., Asymmetric Pentagonal Metal Meshes for Flexible Transparent Electrodes and Heaters. ACS Applied Materials \& Interfaces 2017, 9 (5), 4932-4940.

8. Bae, J. J.; Lim, S. C.; Han, G. H.; Jo, Y. W.; Doung, D. L.; Kim, E. S.; Chae, S. J.; Huy, T. 
Q.; Van Luan, N.; Lee, Y. H., Heat Dissipation of Transparent Graphene Defoggers. Advanced Functional Materials 2012, 22 (22), 4819-4826.

9. Lee, J. G.; Lee, J. H.; An, S.; Kim, D. Y.; Kim, T. G.; Al-Deyab, S. S.; Yarin, A. L.; Yoon, S. S., Highly flexible, stretchable, wearable, patternable and transparent heaters on complex 3D surfaces formed from supersonically sprayed silver nanowires. Journal of Materials Chemistry $A$ 2017, 5 (14), 6677-6685.

10. Li, P.; Ma, J.; Xu, H.; Xue, X.; Liu, Y., Highly stable copper wire/alumina/polyimide composite films for stretchable and transparent heaters. Journal of Materials Chemistry C 2016, $4(16), 3581-3591$.

11. Gupta, R.; Kulkarni, G. U., Holistic Method for Evaluating Large Area Transparent Conducting Electrodes. ACS Applied Materials \& Interfaces 2013, 5 (3), 730-736.

12. Xie, S.; Li, T.; Xu, Z.; Wang, Y.; Liu, X.; Guo, W., A high-response transparent heater based on a CuS nanosheet film with superior mechanical flexibility and chemical stability. Nanoscale 2018, 10 (14), 6531-6538.

13. Kumar, A.; Zhou, C., The Race To Replace Tin-Doped Indium Oxide: Which Material Will Win? ACS Nano 2010, 4 (1), 11-14.

14. Sun, W. J.; Xu, L.; Jia, L. C.; Zhou, C. G.; Xiang, Y.; Yin, R. H.; Yan, D. X.; Tang, J. H.; Li, Z. M., Highly conductive and stretchable carbon nanotube/thermoplastic polyurethane composite for wearable heater. Composites Science and Technology 2019, 181, 107695.

15. Bustillos, J.; Zhang, C.; Boesl, B.; Agarwal, A., Three-Dimensional Graphene FoamPolymer Composite with Superior Deicing Efficiency and Strength. ACS Applied Materials \& Interfaces 2018, 10 (5), 5022-5029.

16. Zhou, R.; Li, P.; Fan, Z.; Du, D.; Ouyang, J., Stretchable heaters with composites of an intrinsically conductive polymer, reduced graphene oxide and an elastomer for wearable thermotherapy. Journal of Materials Chemistry C 2017, 5 (6), 1544-1551. 
17. Zhan, Y. H.; Lago, E.; Santillo, C.; Castillo, A. E. D.; Hao, S.; Buonocore, G. G.; Chen, Z. M.; Xia, H. S.; Lavorgna, M.; Bonaccorso, F., An anisotropic layer-by-layer carbon nanotube/boron nitride/rubber composite and its application in electromagnetic shielding. Nanoscale 2020, 12 (14), 7782-7791.

18. Zhou, B.; Han, X.; Li, L.; Feng, Y.; Fang, T.; Zheng, G.; Wang, B.; Dai, K.; Liu, C.; Shen, C., Ultrathin, flexible transparent Joule heater with fast response time based on single-walled carbon nanotubes/poly(vinyl alcohol) film. Composites Science and Technology 2019, 183, 107796.

19. Ning, W.; Wang, Z.; Liu, P.; Zhou, D.; Yang, S.; Wang, J.; Li, Q.; Fan, S.; Jiang, K., Multifunctional super-aligned carbon nanotube/polyimide composite film heaters and actuators. Carbon 2018, 139, 1136-1143.

20. Wang, Y.; Li, W.; Zhou, Y.; Jiang, L.; Ma, J.; Chen, S.; Jerrams, S.; Zhou, F., Fabrication of high-performance wearable strain sensors by using CNTs-coated electrospun polyurethane nanofibers. J. Mater. Sci. 2020, 55 (26), 12592-12606.

21. Hazarika, A.; Deka, B. K.; Kim, D.; Jeong, H. E.; Park, Y. B.; Park, H. W., Woven Kevlar Fiber/Polydimethylsiloxane/Reduced Graphene Oxide Composite-Based Personal Thermal Management with Freestanding $\mathrm{Cu}-\mathrm{Ni}$ Core-Shell Nanowires. Nano Letters 2018, 18 (11), 6731-6739.

22. Zhang, Q.; Yu, Y.; Yang, K.; Zhang, B.; Zhao, K.; Xiong, G.; Zhang, X., Mechanically robust and electrically conductive graphene-paper/glass-fibers/epoxy composites for stimuliresponsive sensors and Joule heating deicers. Carbon 2017, 124, 296-307.

23. Liang, L. Y.; Xu, P. H.; Wang, Y. F.; Shang, Y.; Ma, J. M.; Su, F. M.; Feng, Y. Z.; He, C. G.; Wang, Y. M.; Liu, C. T., Flexible polyvinylidene fluoride film with alternating oriented graphene/Ni nanochains for electromagnetic interference shielding and thermal management. Chemical Engineering Journal 2020, 395, 10. 
24. Li, W.; Zhou, Y.; Wang, Y.; Li, Y.; Jiang, L.; Ma, J.; Chen, S., Highly Stretchable and Sensitive SBS/Graphene Composite Fiber for Strain Sensors. Macromolecular Materials and Engineering 2020, 305 (3), 1900736.

25. Wang, Q. W.; Zhang, H. B.; Liu, J.; Zhao, S.; Xie, X.; Liu, L.; Yang, R.; Koratkar, N.; Yu, Z. Z., Multifunctional and Water-Resistant MXene-Decorated Polyester Textiles with Outstanding Electromagnetic Interference Shielding and Joule Heating Performances. Advanced Functional Materials 2019, 29 (7), 1806819.

26. Zhao, X.; Wang, L. Y.; Tang, C. Y.; Zha, X. J.; Liu, Y.; Su, B. H.; Ke, K.; Bao, R. Y.; Yang, M. B.; Yang, W., Smart Ti3C2Tx MXene Fabric with Fast Humidity Response and Joule Heating for Healthcare and Medical Therapy Applications. ACS nano 2020, 14, 8793-8805.

27. Li, L.; Cao, Y.; Liu, X.; Wang, J.; Yang, Y.; Wang, W., Multifunctional MXene-Based Fireproof Electromagnetic Shielding Films with Exceptional Anisotropic Heat Dissipation Capability and Joule Heating Performance. ACS Applied Materials \& Interfaces 2020, 12 (24), 27350-27360.

28. Zhou, Y.; Sun, Z.; Jiang, L.; Chen, S.; Ma, J.; Zhou, F., Flexible and conductive metaaramid fiber paper with high thermal and chemical stability for electromagnetic interference shielding. Applied Surface Science 2020, 533, 147431.

29. Lan, W.; Chen, Y.; Yang, Z.; Han, W.; Zhou, J.; Zhang, Y.; Wang, J.; Tang, G.; Wei, Y.; Dou, W.; Su, Q.; Xie, E., Ultraflexible Transparent Film Heater Made of Ag Nanowire/PVA Composite for Rapid-Response Thermotherapy Pads. ACS Applied Materials \& Interfaces 2017, $9(7), 6644-6651$.

30. Pyo, K. H.; Kim, J. W., Transparent and mechanically robust flexible heater based on compositing of Ag nanowires and conductive polymer. Composites Science and Technology 2016, 133, 7-14.

31. Wang, L.; Wang, D.; Wu, Z. F.; Luo, J. C.; Huang, X. W.; Gao, Q.; Lai, X. J.; Tang, L. C.; 
Xue, H. G.; Gao, J. F., Self-Derived Superhydrophobic and Multifunctional Polymer Sponge Composite with Excellent Joule Heating and Photothermal Performance for Strain/Pressure Sensors. Acs Applied Materials \& Interfaces 2020, 12 (11), 13316-13326.

32. Liang, C.; Ruan, K.; Zhang, Y.; Gu, J., Multifunctional Flexible Electromagnetic Interference Shielding Silver Nanowires/Cellulose Films with Excellent Thermal Management and Joule Heating Performances. ACS Applied Materials \& Interfaces 2020, 12 (15), 18023 18031.

33. Wu, L.; Wang, L.; Guo, Z.; Luo, J.; Xue, H.; Gao, J., Durable and Multifunctional Superhydrophobic Coatings with Excellent Joule Heating and Electromagnetic Interference Shielding Performance for Flexible Sensing Electronics. ACS Applied Materials \& Interfaces 2019, $11(37), 34338-34347$.

34. Cheng, Y.; Zhang, H.; Wang, R.; Wang, X.; Zhai, H.; Wang, T.; Jin, Q.; Sun, J., Highly Stretchable and Conductive Copper Nanowire Based Fibers with Hierarchical Structure for Wearable Heaters. ACS Applied Materials \& Interfaces 2016, 8 (48), 32925-32933.

35. Wang, L.; Wang, D.; Wu, Z.; Luo, J.; Huang, X.; Gao, Q.; Lai, X.; Tang, L. C.; Xue, H.; Gao, J., Self-Derived Superhydrophobic and Multifunctional Polymer Sponge Composite with Excellent Joule Heating and Photothermal Performance for Strain/Pressure Sensors. ACS Applied Materials \& Interfaces 2020, 12 (11), 13316-13326.

36. Sun, Z.; Zhou, Y.; Li, W.; Chen, S.; You, S.; Ma, J., Preparation of Silver-Plated ParaAramid Fiber by Employing Low-Temperature Oxygen Plasma Treatment and Dopamine Functionalization. Coatings 2019, 9 (10), 599.

37. Liu, C.; Liu, J.; Ning, X.; Chen, S.; Liu, Z.; Jiang, S.; Miao, D., The Effect of Polydopamine on an Ag-Coated Polypropylene Nonwoven Fabric. Polymers 2019, 11 (4), 627.

38. Shi, Y. D.; Li, J.; Tan, Y. J.; Chen, Y. F.; Wang, M., Percolation behavior of electromagnetic interference shielding in polymer/multi-walled carbon nanotube 
nanocomposites. Composites Science and Technology 2019, 170, 70-76.

39. Nallabothula, H.; Bhattacharjee, Y.; Samantara, L.; Bose, S., Processing-Mediated Different States of Dispersion of Multiwalled Carbon Nanotubes in PDMS Nanocomposites Influence EMI Shielding Performance. ACS Omega 2019, 4 (1), 1781-1790.

40. Yang, X.; Fan, S.; Li, Y.; Guo, Y.; Li, Y.; Ruan, K.; Zhang, S.; Zhang, J.; Kong, J.; Gu, J., Synchronously improved electromagnetic interference shielding and thermal conductivity for epoxy nanocomposites by constructing 3D copper nanowires/thermally annealed graphene aerogel framework. Composites Part A: Applied Science and Manufacturing 2020, 128, 105670. 41. Wang, L.; Song, P.; Lin, C. T.; Kong, J.; Gu, J. W., 3D Shapeable, Superior Electrically Conductive Cellulose Nanofibers/Ti3C2Tx MXene Aerogels/Epoxy Nanocomposites for Promising EMI Shielding. Research 2020, 2020, 12.

42. Liu, L. X.; Chen, W.; Zhang, H. B.; Wang, Q. W.; Guan, F. L.; Yu, Z. Z., Flexible and Multifunctional Silk Textiles with Biomimetic Leaf-Like MXene/Silver Nanowire Nanostructures for Electromagnetic Interference Shielding, Humidity Monitoring, and SelfDerived Hydrophobicity. Advanced Functional Materials 2019, 29 (44), 1905197.

43. Jiang, L.; Zhou, Y.; Guo, Y.; Jiang, Z.; Chen, S.; Ma, J., Preparation of silver nanoparticle functionalized polyamide fibers with antimicrobial activity and electrical conductivity. Journal of Applied Polymer Science 136 (22), 47584.

44. Ma, Z.; Kang, S.; Ma, J.; Shao, L.; Zhang, Y.; Liu, C.; Wei, A.; Xiang, X.; Wei, L.; Gu, J., Ultraflexible and Mechanically Strong Double-Layered Aramid Nanofiber-Ti3C2Tx MXene/Silver Nanowire Nanocomposite Papers for High-Performance Electromagnetic Interference Shielding. ACS Nano 2020, 14 (7), 8368-8382.

45. Luo, J.; Huo, L.; Wang, L.; Huang, X.; Li, J.; Guo, Z.; Gao, Q.; Hu, M.; Xue, H.; Gao, J., Superhydrophobic and multi-responsive fabric composite with excellent electro-photo-thermal effect and electromagnetic interference shielding performance. Chemical Engineering Journal 
2020, 391, 123537.

46. Zhang, X.; Jiang, S. X.; Cai, M.; Zhao, H. M.; Pan, F. K.; Miao, D. G.; Ning, X., Magnetron sputtering deposition of $\mathrm{Ag} / \mathrm{Ag} 2 \mathrm{O}$ bilayer films for highly efficient color generation on fabrics. Ceram. Int. 2020, 46 (9), 13342-13349.

47. Li, X.; Tang, C.; Wang, J.; Tian, W.; Hu, D., Analysis and mechanism of adsorption of naphthenic mineral oil, water, formic acid, carbon dioxide, and methane on meta-aramid insulation paper. J. Mater. Sci. 2019, 54 (11), 8556-8570.

48. Zille, A.; Fernandes, M. M.; Francesko, A.; Tzanov, T.; Fernandes, M.; Oliveira, F. R.; Almeida, L.; Amorim, T.; Carneiro, N.; Esteves, M. F.; Souto, A. P., Size and Aging Effects on Antimicrobial Efficiency of Silver Nanoparticles Coated on Polyamide Fabrics Activated by Atmospheric DBD Plasma. ACS Applied Materials \& Interfaces 2015, 7 (25), 13731-13744.

49. Wang, W.; Li, R.; Tian, M.; Liu, L.; Zou, H.; Zhao, X.; Zhang, L., Surface Silverized MetaAramid Fibers Prepared by Bio-inspired Poly(dopamine) Functionalization. ACS Applied Materials \& Interfaces 2013, 5 (6), 2062-2069.

50. Lu, Z.; Hu, W.; Xie, F.; Hao, Y.; Liu, G., Argon low-temperature plasma modification of chopped aramid fiber and its effect on paper performance of aramid sheets. 2017, 134 (34), 45215.

51. Hwang, Y. J.; Qiu, Y.; Zhang, C.; Jarrard, B.; Stedeford, R.; Tsai, J.; Park, Y. C.; McCord, M., Effects of atmospheric pressure helium/air plasma treatment on adhesion and mechanical properties of aramid fibers. Journal of Adhesion Science and Technology 2003, 17 (6), 847860.

52. Choi, S.; Park, J.; Hyun, W.; Kim, J.; Kim, J.; Lee, Y. B.; Song, C.; Hwang, H. J.; Kim, J. H.; Hyeon, T.; Kim, D.-H., Stretchable Heater Using Ligand-Exchanged Silver Nanowire Nanocomposite for Wearable Articular Thermotherapy. ACS Nano 2015, 9 (6), 6626-6633.

53. Hsu, P. C.; Liu, X.; Liu, C.; Xie, X.; Lee, H. R.; Welch, A. J.; Zhao, T.; Cui, Y., Personal 
Thermal Management by Metallic Nanowire-Coated Textile. Nano Letters 2015, 15 (1), 365371.

54. Kim, T.; Kim, Y. W.; Lee, H. S.; Kim, H.; Yang, W. S.; Suh, K. S., Uniformly Interconnected Silver-Nanowire Networks for Transparent Film Heaters. 2013, 23 (10), 12501255.

55. Gao, J.; Luo, J.; Wang, L.; Huang, X.; Wang, H.; Song, X.; Hu, M.; Tang, L. C.; Xue, H., Flexible, superhydrophobic and highly conductive composite based on non-woven polypropylene fabric for electromagnetic interference shielding. Chemical Engineering Journal 2019, 364, 493-502.

56. Lin, L.; Wang, L.; Li, B.; Luo, J.; Huang, X.; Gao, Q.; Xue, H.; Gao, J., Dual conductive network enabled superhydrophobic and high performance strain sensors with outstanding electro-thermal performance and extremely high gauge factors. Chemical Engineering Journal 2020, 385, 123391. 


\section{Table of Contents}

AgNPs coated meta-aramid fiber paper

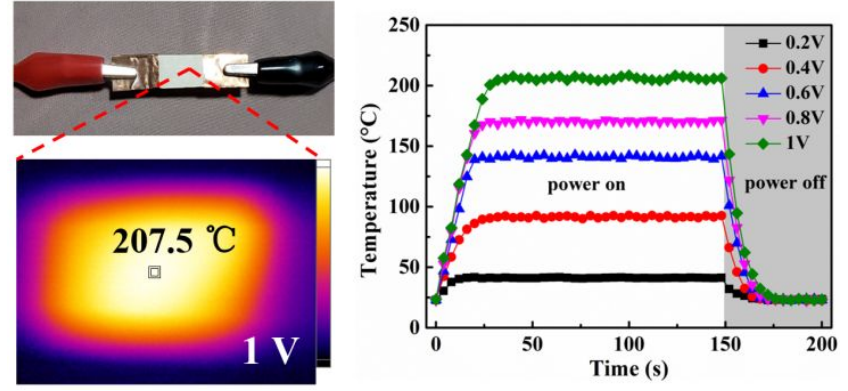

